Radial and Nonradial Pulsations as Probes of Stellar Physics

ASP Conference Series, Vol. 259, 2002

C. Aerts, T.R. Bedding, \& J. Christensen-Dalsgaard, eds.

\title{
Stellar Parameters of the Post-AGB Star HD 56126
}

\author{
H. Le Coroller ${ }^{1}$, D. Gillet \\ Observatoire de Haute-Provence (CNRS), France
}

\author{
A.B. Fokin \\ Institute of Astronomy, Russian Academy of Sciences, Russia
}

A. Lèbre

GRAAL, Université Montpellier II \& CNRS, France

Abstract. We discuss the stellar parameters $\left(M, L, T_{\text {eff }}\right)$ of a postAGB star, HD 56126, deduced from observations and non-linear radiative pulsation models. We show that pure radiative pulsation models are in contrast with stellar evolution.

\section{Stellar parameters of HD 56126}

HD 56126 is a typical carbon-rich post-AGB star with a detached dust shell and IR emission (Meixner et al., 1997; Jura et al., 2000). This star is pulsating with a period of $36.8 \pm 0.2$ days, a photometric amplitude around $0.15 \mathrm{mag}$, and a photospheric velocity variation of about $15 \mathrm{~km} \mathrm{~s}^{-1}$ (Barthès et al., 2000). To reproduce these observational features we have looked for a non-linear solution through several grids of models (Fokin et al., 2001). For $M<0.7 M_{\odot}$ and any $L$ and $T_{\text {eff }}$ the photometric amplitude is too large compared to the observations (Fig. 1). We do find a consistent solution with the observations for stellar parameters : $M=0.8 M_{\odot}, L=6000-7000 L_{\odot}$, and $T_{\text {eff }} \leq 5850 \mathrm{~K}$.

\section{Discussion and future work}

We found that HD 56126 could pulsate in the first overtone of a radial mode with the following stellar parameters: $M=0.8 M_{\odot}, L=6000-7000 L_{\odot}$, and $T_{\text {eff }} \leq$ $5850 \mathrm{~K}$. Our result seems to be in contradiction with the stellar evolution. Indeed the mass-luminosity relations (e.g. Herwig et al., 1998) point to a luminosity higher than $16000 L_{\odot}$ for a mass of $0.8 M_{\odot}$. Moreover, low mass stars under $0.7 M_{\odot}$ are in better agreement with the evolutionary status of HD 56126 which is a metal deficient Pop.II star.

\footnotetext{
1e-mail: coroller@obs-hp.fr
} 

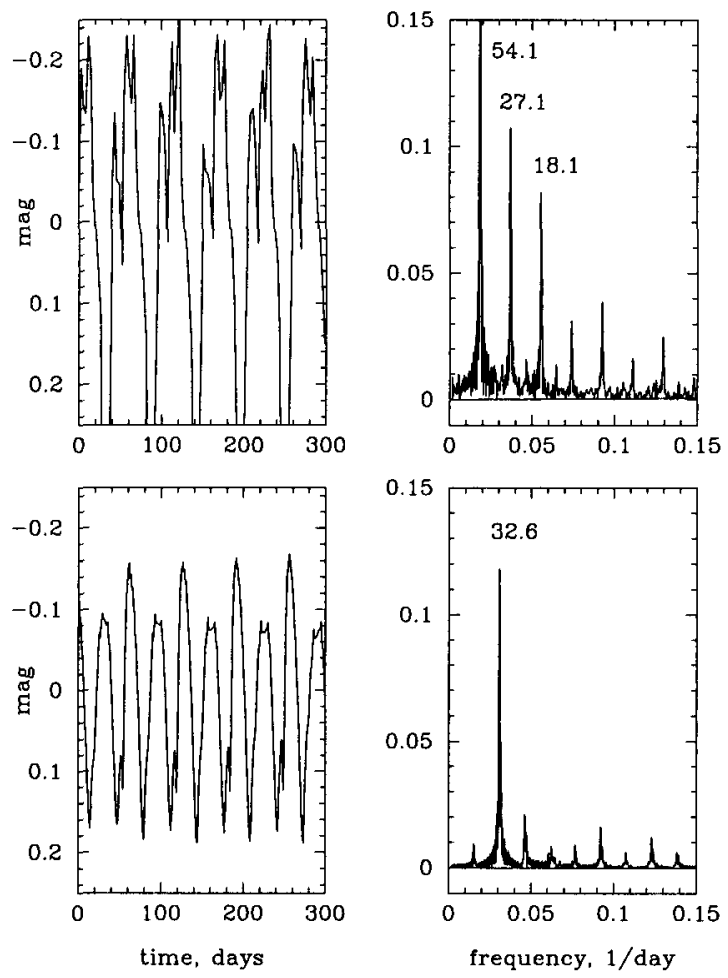

Figure 1. Light curves and power spectra for 2 pulsation models: $L=6000 \mathrm{~L}_{\odot}, T_{\text {eff }}=5750 \mathrm{~K}, M=0.6 \mathrm{M}_{\odot}$ (upper) and $M=0.8 \mathrm{M}_{\odot}$ (lower).

We used a pulsation model that does not take into account the convection, which could change the pulsational behavior of the model. Moreover, we used a Lagrangian code while models with an adaptative grid code are more suited to resolve accurately the hydrogen ionization zone. Additionally, the dependence of the mass-luminosity relation on the metallicity is still poorly known and it could partly be responsible for the discrepancy between our results and the stellar evolution relation. We will elaborate on these points in future work.

\section{References}

Barthès, D., Lèbre, A., Gillet, D., \& Mauron, N. 2000, A\&A, 359, 168

Fokin, A.B., Lèbre, A., Le Coroller, H., \& Gillet, D. 2001, A\&A, in press Herwig, F., Schönberner, D., \& Blöcker, T. 1998, A\&A, 340, L43

Jura, M., Chen C., \& Werner M. W. 2000, ApJ, 544, L141

Meixner, M., et al. 1997, ApJ, 482, 897 